\title{
Références
}

\section{Le feedback (ou rétro-action) : un élément essentiel de l'intervention pédagogique en milieu clinique}

Heather V. OBRIEN, ${ }^{*}$ Meredith B. MARKS, ${ }^{*}$ Bernard CHARLIN* *

M esagaes clés - Pour les éducateurs, donner du feedback aux étudiants représenteune habiletécomplexe mais nécessaire. - II y a feedback lorsque l'éudiant reçoit de l'information concernant une de ses actions et qu'il utilise cette information pour modifier son approche de la tâche. - Sans feedback l'apprentissage est retardé; les éudiants peuvent sancrer dans de mauvai ses habitudes et abandonner sans le savoir des modal ités d'action pertinentes. - Un feedback efficace est donné en temps voulu. II porte sur des comportements spécifiques et remé diables, il impliquel'étudiant et il identifie à la fois lesforces et lesfai blesses. II et objectif sans porter de jugement de valeur et il offre des suggestions de changement. - D onner du feedback implique toujours un respect de l'étudiant. - L e feedback n'est pas une habileté innée. II peut sapprendre. Avec un peu de feedback vos habiletés de feedback peuvent saméliorer.

Mots clés Feedback, éducation médicale, méthodes d'enseignement.

Key learning points - Presenting feedback to learners is a complex but necessary skill for educators. - Feedback occurs when a learner is provided with information regarding an action and then uses that information to modify their approach to a task. - Without feedback learning is delayed; learners may refine bad habits and unknowingly abandon effective practices. - Effective feedback is provided in a timely manner, focuses on specific behaviours that are remediable, involves the learner, identifies both strengths and weaknesses, is objective and non-judgmental, and offers suggesti ons for change. - When providing feedback, always maintain respect for the learner. - F edback is not an innate skill ; it can be learned. With a little feedback your feedback skills can improve.

Keywords Feedback; medical education; teaching methods.

Pédagogie M édicale $2003 ; 4:$ : 184-191

\section{Introduction}

Comment informons nous au mieux les étudiants à propos dela qualitédeleur performanceclinique et comment les motivons nous pour qu'ils l'améliorent en permanence? D ansune perspective éducationnelle, la pratique de la médecine est souvent vue comme étant l'application d'un ensemble de savoirs, d'habiletés et d'attitudes.
Les interrelations de ces trois domaines sont complexes. De ce fait, pour analyser et optimiser les pratiques cliniques d'un étudiant en formation, il est nécessaire de recourir à des stratégies et à des techniques d'intervention pédagogique variées. L'observation directe par un enseignant d'un étudiant en situation de pratique clinique réelle offre l'opportunité d'apprécier les forces et les fai-

\footnotetext{
* Université d'Qtawa* * Université de Montréal

Correspondance: Heather V. O'Brien - Département d'anesthésiologie - Université d'Ottawa - Ontario - Canada, mailto : Hobrien@cheo.on.ca
} 
blesses de l'étudiant et de lui retourner cette information. Cette capacité de donner du feedback (ou rétro-action) est une des composantes essentielles de l'éducation en milieu clinique.

Les activités de soins spécifiques au lit du malade constituent le support principal de la formation en milieu clinique. $D$ ans cet environnement les enjeux sont importants, tant pour les enseignants que pour les étudiants. Les soins aux patients sont au centre de notre mission et la sécurité du patient doit toujours être notre priorité. Les étudiants apprennent sur un mode expérientiel en prenant des décisions et en intervenant, tout en observant les conséquences de leurs actions. IIs ont certes des capacités d'auto-évaluation, mais ils ont soif de feedback pour être rassurés et, le cas échéant, réorientés quant à leur progression dans la bonne direction.

Chacun d'entre nous enseigne dans des microcosmes au sein de l'environnement académique. II n'est pas facile de retrouver dans la littérature pédagogique les informations répondant defaçon pertinenteet spécifiqueaux problèmes singuliers que nous avons à résoudre. Les caractéristiques des étudiants, les méthodes d'enseignement employées et les contextes professionnels varient considérablement. II arrive que nous travaillions à côté d'un étudiant et en interaction directe avec lui pendant une période prolongée; il arrive égal ement que nous interagi ssions ponctuellement avec de nombreux apprenants, en n'ayant que de rares occasions de travail prolongé avec chacun d'entre eux. Ces conditions variables de la pratique éducative en contexte clinique créent des difficultés pour donner du feedback. Cependant des principes généraux prenant en compte les exigences d'un feedback efficace peuvent être aisément adaptés à pratiquement toutes les situations d'enseignement.

L'objectif de cet article est de mettre l'accent sur la valeur pédagogique du feedback donné sur une base régulière, de présenter les composantes du feedback efficace, telles qu'elles ont été développées et codifiées dans la littérature et, sur la base des données probantes actuellement disponibles, deformuler des recommandations pour donner un feedback efficace.

\section{Qu'est-ce que le feedback ? Pourquoi est-il important ?}

Le concept de feedback a été énoncé pour la première fois dans les années 40 . Le terme désignait un processus de recueil d'informations concernant lemouvement de fusées et la transmission en retour de cette information, dans un format utilisable, vers le système de programmation des trajectoires ultérieures du projectile. Apparu dans le champ de l'ingénierie, le concept fut ensuite extrapolé pour devenir le fondement d'une science plus large, la cybernétique'. D ans les années 70 , on assista à la transposition du concept de feedback dans des domaines très divers, incluant le monde des affaires et celui de la médecine $e^{2.5}$. La notion de boucle courte, au sein de laquelle se succèdent l'action, I'observation, l'interprétation et la redirection, caractérise fondamentalement le processus de feedback, qui peut être appliquéà tout scénario dans lequel le but viséest un changement de comportement.

En éducation médicale, le terme feedback fait référence à un message spécifique, basé sur l'observation de l'étudiant en train d'effectuer une tâche professionnelle, fourni par l'enseignant et communiqué à l'étudiant dans l'intention del'informer et delui offrir uneopportunité pour améliorer sa performance dans les tâches ultérieures. Le feedback résulte de la fourniture à un étudiant d'un éclairage sur ce qu'il ou elle vient d'effectuer, ainsi que sur les consé quences de ses actions ${ }^{6}$. Le feedback consiste à observer et re-diriger, dans un cycle continu d'essais-erreurs. L'étudiant entreprend une action, dans une situation donnée, avec l'intention d'obtenir un résultat particulier (faciliter la communication, recueillir de l'information diagnostique, développer un diagnostic différentiel, mettreen route un traitement, etc.). Le rôle de l'enseignant consiste à fournir à l'éudiant un point de vue objectif à l'égard de ce qu'ont été ses actions réelles et des conséquences prévisibles de ces actions ${ }^{6}$. L'introspection développée par l'étudiant, guidée autant que nécessaire par l'enseignant, l'aide à comprendre comment ses futures actions devront être modifiées pour produire les résultats désirés.

$D$ ans cet article, le terme feedback désigne le feedback formatif, qui diffère du feedback sommatif. Le feedback formatif, tout commel'entrainement sportif, est fourni tout au long du cheminement vers le but. A l'opposé, le feedback sommatif est fourni après l'action. II est utilisé aux fins d'attribution de notes, de rang de mérite, de certification ou pour délivrer un droit d'exercice. Utilisé isolément, le feedback sommatif prive l'étudiant de l'opportunité de modifier ses pratiques dans le cadre d'un processus continu.

Les termes de feedback efficace et de feedback inefficace font référence à la façon dont le message est transmis. Le feedback efficace (le «bon » feedback, le feedback bénéfique) guide l'étudiant vers le but désiré, tandis que le feedback 


\section{Références}

inefficace (le «mauvais » feedback, le feedback délétère) peut produire l'effet inverse. Ces termes sont différents de ceux de feedback positif (ou de renforcement) et de feedback négatif (ou correctif) qui eux concernent, dans le processus du feedback, les conséquences du comportement del'étudiant.

Le feedback efficace procure de nombreux bénéfices, tant à l'étudiant et qu'à l'enseignant. II aide l'étudiant à définir ses attentes, à évaluer ses apprentissages, à agir sous supervision et, en fin de compte, à améliorer sa performance. Le fait de générer du feedback bénéficie aussi àl'enseignant en lui permettant de modifier son style et ses contenus d'enseignement pour sadapter aux besoins individuels des étudiants. En conséquence, le feedback contribue à créer un climat de préoccupation et d'intérêt sincères envers le développement de l'étudiant. Le bon feedback incite l'étudiant à se sentir responsable et fier de ce qu'il sait et de ce qu'il sait bien faire, à entreprendre les actions nécessaires pour combler les lacunes de ses connaissances et à approfondir la compréhension de son champ d'étude. La capacité à donner adéquatement du fedback a été identifiée comme étant une des caractéristiques des enseignants cliniciens experts?.

A l'inverse, l'incapacité à procurer du feedback peut avoir des conséquences négatives. $\mathrm{N}$ os étudiants peuvent ne pas percevoir leurs erreurs, construire des conceptions erronées et développer de mauvaises habitudes. Leur progression est ralentie quand leurs faiblesses ne sont pas identifiées en temps voulu. Les étudiants peuvent aussi ne pas prendre conscience de la qualité de leurs réalisations et abandonner ainsi sanslesavoir desfaçons defaireefficaces. Sans feedback, les étudiants risquent peu à peu de se satisfaire de critères moins objectifs et moins exigeants et graduellement de rejeter délibérément toute source d'évaluation externe, alors même qu'ilsne sont pas encore devenus autonomes.

Les étudiants ont souvent du mal à reconnaître que du feedback leur a été donné, surtout si le feedback n'a pas été identifié explicitement comme tel par le superviseur'. II a été montré que les superviseurs perçoivent plus facilement que leurs étudiants qu'un feedback spécifique, bien conçu, a été donné en temps voulu9. Au sein d'un groupe de résidents de médecine interne interrogés par questionnaire quant à l'existence d'un feedback, $80 \%$ d'entre eux ont rapportéqu'ils n'avaient jamais ou peu fréquemment reçu du feedback alors même qu'ils en avaient reçu régulière ment ${ }^{10}$. Pour autant, il faut admettre qu'un feedback n'est pas toujours donné régulièrement par les superviseurs cliniques, ${ }^{7}$. C eci n'est pas propre au domaine dela formation médicale. $D$ es problèmes similaires ont été décrits dans d'autres discipline ${ }^{11,12}$. Les superviseurs, que ce soit dans les milieux cliniques ou non, se sentent volontiers mal à l'aise pour faire face aux émotions qui peuvent apparaitre lorsque le feedback est donné. La plupart des enseignants appréhendent de dire à leurs étudiants que leurs pratiques sont incorrectes, de peur de heurter leur sensibilité. Ils se sentent d'ailleurs souvent tout aussi maladroits pour féliciter leurs étudiants. Afin de ne pas se trouver confrontés eux-mêmes aux conséquences de la charge affective induite par le feedback, bien des superviseurs évitent inconsciemment d'affronter les exigences requises pour donner du feedback et choisissent de ne pas en donner du tout ${ }^{11,13}$.

À l'opposé, certains enseignants formulent des critiques ou des louanges si excessives qu'elles en deviennent non cré dibles et sont de ce fait rejetées par les étudiants. Par ailleurs même un feedback bien intentionné, s'il est mal donné, peut amener l'étudiant à penser qu'un jugement de valeur est porté sur sa personnalité ou à croire que ses pensées, opinions et contributions ont été négligées ou rejetées.

L'art de donner un feedback efficace n'est pas inné mais peut en revanche sapprendre. Comme pour la plupart des compétences d'enseignement, le confort découle de la maîtrise d'habiletés et la maîtrise requiert une pratique régulière

\section{Recommandations pour la délivrance d'un feedback}

En 1983, Jack Ende a publié dans le JAM A son article princeps intitulé «Feedback in Clinical M edical Education $»^{6}$. Vingt ans plus tard il demeure difficile de trouver un article important sur le thème du feedback qui ne cite pas ce travail. $D$ ans son article il définit le rôle du feedback en éducation médicale et émet un ensemble clair de recommandations pour donner un feedback efficace. II faut noter qu'en 1983, Ende ne fournissait pas de preuve pour étayer ses recommandations. Les éléments de preuve, issus des travaux d'autres auteurs ${ }^{11,12}$, sont arrivés plus tard. L'efficacité des techniques de feedback proposées dans la littérature a été démontrée dans des situations réelles. Par exemple, il a été demandé aux participants d'un atelier consacré aux habiletés de communication d'identifier des exemples de feedback utile ou inutile reçus pendant la formation ${ }^{14}$. Les caractéristiques de l'un et l'autre ont été 
éval uées à la fois quantitativement et qualitativement. Cette étude concernait des enseignants cliniciens qu'on pouvait supposer plus réceptifs que d'autres apprenants à recevoir du feedback. Cependant, ilsétaient clairement placés dans un rôle d'apprenant et étai ent activement engagés dans l'acquisition de nouvelles habiletés. Le feedback évalué dans cette étude était donc bien fourni dans un contexte d'apprentissage authentique. Les résultats de l'étude accréditent tout à fait l'efficacité des techniques de feedback identifiées dans la littérature et que nous résumons ci-dessous.

Lorsqu'on songe à donner du feedback, six questions méritent d'être prises en considération :

\section{Suis-je la personne appropriée pour donner ce feedback?}

Le feedback peut être fourni par quiconque ayant l'expérience de la situation d'apprentissage (et de ce qu'elle représente comme défi pédagogique) et ayant été en position d'observer attentivement la performance de l'étudiant. L'information sera par ailleurs d'autant mieux reçue par l'étudiant qu'il vous percevra comme étant une source fiable ayant des intentions sincères envers lui. II n'est pas acceptable de fournir du feedback hors de votre domaine d'expertise ou lorsque vous n'êtes pas directement impliqué dans la situation. En revanche, toute personne qui contribue à l'évaluation sommative de l'étudiant a la responsabilité de veiller à ce qu'un feedback formatif appropriésoit fourni en quantitéet qualitésuffisantependant la périodequi précède cette évaluation.

\section{Sur quel point particulier le feedback doit-il porter?}

Le feedback doit se concentrer particulièrement sur les comportements de l'étudiant qui sont observables et qui peuvent être comparés à des buts ou des standards de pratique bien établis. Idéalement le feedback est basé sur ce que vous avez observé directement vous-même. II faut être certain que les observations et les informations utilisées pour donner le feedback sont valides et qu'elles peuvent être comparées à des standards de pratique appropriés. Votre feedback devrait porter exclusivement sur les comportements qui sont remédiables. II n'y a aucun intérêt à identifier des détails qui ne peuvent être modifiés. Par exemple, en discutant la façon dont un étudiant communique avec les patients, il est inutile de faire des commen- taires sur le caractère haut perché de la voix de l'étudiant. Cen'est pas quelque chose qu'il peut changer. Cette information va contrarier l'étudiant et le rendre moins susceptible d'accepter des suggestions qui pourraient avoir un impact réel sur ses pratiques cliniques.

Lefeedback correctif identifie un écart entre des comportements existants et des comportements attendus. Le feedback positif souligne les pratiques qui favorisent le but recherché. Les deux modalités de feedback permettent d'améliorer la performance de l'étudiant en contribuant à combler cet écart. II peut également être utile de comparer la performance de l'étudiant avec ses performances passées dans cette tâche, avec sa performance dans d'autres tâches ou avec la performance de ses pairs ${ }^{15}$.

II est souhaitable de limiter votre message aux faits et aux résultats réellement observés, sans présumer des intentions de l'étudiant. II est, par exemple, fréquent de présumer que si un étudiant a négligé une donnée clinique importante c'est parce qu'il a oublié de la rechercher. En fait, la raison réelle pouvait être que l'étudiant n'avait pas les connaissances et les habiletés pour évaluer adéquatement ce patient. II n'est pas correct de postuler arbitrairement la cause d'un comportement observé. II convient plutôt de mettre l'accent sur la description de ce qui a été observé, pour explorer ensuite, avec l'étudiant, les raisons de son action et de ses conséquences.

Les commentaires généraux concernant l'intelligence de l'étudiant, ses qualités d'organisation, ses compétences ou ses attitudes sont rarement utiles sils ne sont pas rattachés à des actions ou à des événements spécifiques. D ela même façon, l'expression de compliments globaux est beaucoup moins utile que l'identification d'actions qui ont eu des conséquences positives et qui méritent un renforcement. Les traits de personnalité ne sont pas habituellement des objets de feedback, sauf sils setraduisent en comportements qui sont corrigeables ou qui méritent d'être renforcés.

\section{0 ù et quand dois-je offrir du feedback ?}

Le feedback efficace fait partie des attentes légitimes de nos étudiants. Idéalement, les étudiants devrai ent rechercher les opportunités de feedback et ne pas subir ce dernier comme un rite nécessaire. Le feedback attendu, donné régulièrement, est moins susceptible de provoquer des réactions émotives et a plus de chance d'être perçu comme étant crédible.

Lefeedback devrait être discutéàun moment appropriéde 


\section{Références}

façon à ceque l'enseignant et l'étudiant puissent tous deux se rappel er les détails de la performance analysée et les conséquences. Le feedback doit être donné aussitôt que possible après la période d'observation de l'étudiant. II est efficace quand tous ceux qui sont impliqués se souviennent des détails et avant que de fortes émotions ou une déconnexion de l'événement aient eu le temps de se produire.

Le moment du feedback est donc important. II est cependant des circonstances où il vaut mieux délibérément le différer si l'enseignant et/ou l'étudiant ressentent de fortes émotions. N ous nous souvenons tous d'événements au cours desquels un étudiant a commis une erreur critique pendant une situation stressante, à un moment de surcharge de travail clinique. Q uand l'étudiant fait face à une tâche qu'on ne juge pas soi-même particulièrement compliquée, le risque est plus grand de se sentir insatisfait de l'étudiant. $D$ ans ce genre de situation, nous avons tendance à évaluer la personnalitéde l'étudiant plutôt que ses actions. C'est une situation rarement productive. D e la même façon, si un étudiant est encore émotivement perturbé par un événement qui vient dese produire, il est souhaitable de lui donner du temps pour rassembler ses idées avant de lui offrir vos observations. Attendez donc un peu pour que vos émotions et celles de l'étudiant aient eu le temps de se calmer avant de donner du feedback, mais ne repoussez pas ce dernier indéfiniment.

Le feedback efficace doit être donné dans un environnement favorable. Le superviseur doit être perçu par l'étudiant commeun alliécentré sur le but commun d'améliorer sa performance. II n'est pas nécessaire que l'enseignant se force à apparaitre comme un complice ou un pair, mais la fourniture du feedback devrait refléter la sagesse et l'expérience de l'enseignant, sans accentuer indûment la différence de pouvoir et de statut entre l'enseignant et l'étudiant. Pour de multiples raisons, il y aura toujours des moments et des endroits qui n'apparaitront pas idéaux, dans la perspective de l'un ou l'autre, pour donner du feedback. II devrait toutefois être toujours possible d'offrir à l'étudiant un feedback approfondi, qui tienne compte du contexte émotif de l'étudiant dans une situation donnée.

\section{Comment dois-je m'y prendre?}

II est nécessaire de travailler sur des buts communs en impliquant activement l'étudiant dans son propre feedback. II doit être invitéà donner ses appréciationsà propos de ses actions, de leurs forces et de leurs fai blesses et sur la façon dont il pense pouvoir les améliorer dans la perspective des performances futures. C ette auto-éval uation préalable prépare l'étudiant à recevoir l'évaluation d'un observateur extérieur sur ces mêmes actions. D emandez à l'étudiant comment il perçoit ses actions et leurs consé quences. Soyez préparé à l'écouter avec un esprit ouvert. C'est cemodèle d'ouverture d'esprit que les étudiants doivent incorporer pour pouvoir accepter le feedback.

Le feedback devrait porter sur la performance de l'étudiant par rapport à des buts prédéfinis, tels que les objectifs éducationnels de la période de stage. Les deux parties devraient partager les mêmes buts et valeurs. S'il y a écart àceniveau, il est probable qu'il faudra partager plus que de I'information ; il faudra peut-être même parfois se résoudre à une approche de résolution de conflit. Stritter ${ }^{16}$ suggère que le feedback formatif tout comme le feedback sommatif doivent faire explicitement partie du contrat d'apprentissage initial. Les étudiants devraient savoir qu'ils vont êtreévalués et ilsont le droit desavoir à quel moment exact ils vont l'être.

La quantité de feedback que l'on peut donner à un moment déterminéest limitée. Il y a une limiteau nombre de messages qu'un étudiant peut enregistrer. II est nécessaire de mettre l'accent sur les points les plus significatifs. Si vous donnez régulièrement du feedback, vous aurez d'autres occasions pour formuler d'autres messages.

Ende ${ }^{6}$ ne donne pas de recommandation précise sur la façon d'équilibrer feedback positif et feedback négatif pour optimiser l'impact sur l'étudiant. Un feedback négatif peut avoir l'effet désiré en réduisant les comportements non souhaitables, mais il peut aussi augmenter le niveau d'anxiétéde l'apprenant. Un enseignant qui utilise excessivement le fedback négatif va faire décroître la réceptivité del'appren ant vis-à-vis du feedback ultérieur, érigeant ainsi une barrière à la communication. Un juste équilibre montre à l'apprenant que vous observez autant ses forces que ses faiblesses. Ce faisant, vous l'encouragerez à vous écouter plus attentivement.

Selon Stritter ${ }^{16}$, les commentaires négatifs sont habituellement mieux reçuss'ils sont accompagnés de commentai res positifs. Ses observations l'ont amené à proposer le concept de «feedback sandwich » dans lequel un point de feedback positif est suivi par un point de feedback négatif et ensuite coiffé d'une observation de renforcement positif, afin de terminer la session sur une note positive. $\mathrm{Ce}$ concept de « feedback sandwich » est devenu très populaire. $D$ 'autres auteurs considèrent cependant qu'une attitude aussi systématique devient trop prévisible de la 
part del'étudiant qui, dans ce cas, peut négliger lefeedback positif en raison de son côtéconvenu et se préoccuper uniquement de la tranche négative du sandwich ${ }^{17}$.

Un des concepts utiles de la littérature est celui du compte en banque émotif ${ }^{15}$. En tant qu'enseignant, vous pouvez faire des dépôts sur le compte de l'étudiant en lui offrant du feedback positif de renforcement. Vous êtes alors autorisé à effectuer des retraits sous forme de critique correctrice tout en veillant sur le sol de de votre compte avec I'apprenant. Un solde suffisant vous permettra de faire des retraits isolés, en prenant garde à tout moment à ne pas amener votre compte dans la zone négative.

Le feedback devrait être formulé en utilisant un langage précis et objectif. II devrait se limiter à une évaluation neutre et objective de la performance, dans le but d'amé liorer les habiletés professionnelles. Le feedback ne vise ni à susciter une réponseémotive de l'étudiant, ni à critiquer sa valeur personnelle. Formulé adéquatement, il est purement descriptif et ne juge pas. Si des éléments subjectifs doivent être émis, ils devrai ent l'être en utilisant des phrases du genre «J'ai observé que... » ou «il m'apparaît que... ». C eci permet au récepteur du feedback de se rendre compte que l'impact de son comportement peut être différent decelui qu'il ou elle espérait avoir ou pensait avoir eu, sans avoir lesentiment d'avoir éééjugé.

Cequi manque manifestement dans les recommandations formulées par Ende ${ }^{6}, c^{\prime}$ est la notion que les enseignants donnant du feedback devraient y inclure des suggestions pour permettre une amélioration. Les auteurs ultérieurs ont démontré cette nécessité, ainsi que celle de recommandations de lectures utiles et de propositions concernant ce que l'étudiant devrait essayer la prochaine fois pour mieux faire. Une étude au moins montre clairement que l'inclusion de suggestions de changement est associée à une perception par les apprenants que le feedback est utile $e^{14}$. La littérature suggère donc de se sentir libre d'indiquer des directions, de suggérer des articles à lire, de stimuler, c'est-à-dire d'enseigner, tout simplement !

Lorsque vous et votre étudiant convenez qu'un domaine de pratique requiert des améliorations et que des suggestions correctives sont suggérées, il faut idéalement renseigner l'étudiant quant aux résul tats de ses efforts ultérieurs en assurant un suivi. Ceci permet de s'assurer que l'étudiant corrige ses faiblesses, tout en lui faisant percevoir l'importance que vous placez dans la qualité de son apprentissage clinique.

Suggérer des solutions alternatives pour atteindre les résultats désirés est une manière de donner du feedback négatif d'une façon positive. Par exemple, «Une autre façon de faire cela pourrait avoir été de... ». Le questionnement peut être utilisé comme technique pour amener I'apprenant à réfléchir sur les conséquences de ses actions. Par exemple : «Y a-t-il un effet secondaire de ce médicament qui pourrait rendre son utilisation dangereuse dans cette situation?»

Le superviseur qui donne du feedback devrait toujours vérifier si le message a bien étéémis et compris. Le langage non verbal peut à cet égard être trompeur. Un hochement de tête peut être interprété comme de l'impatience, plutôt que comme un accord et une compréhension. II est donc utile de demander à l'étudiant qui reçoit du feedback de réémettre le message à votre intention, de l'encourager à reconnaitre les comportements qui demandent à être modifiés et de se commettre sur la façon dont il comptes'y prendre et sur son engagement à le faire.

\section{A quelle réponse émotive dois-je m'attendre?}

En dépit des meilleures intentions de l'enseignant et de I'apprenant, tant qu'une certaine expérience n'a pas été accumulée d'un côté comme de l'autre, le feedback est habituell ement donné et reçu avec un certain degré d'anxiété et d'incertitude. II est des situations dans lesquelles il faut une grande dose d'habileté pour fournir un feedback sans en limiter l'efficacitéen engendrant une réaction défensive chez l'apprenant.

Si votre personnal ité vous inciteà aller rapidement au fond des choses, méfiez-vous. Une critique pertinente et précise n'autorise pas pour autant à prononcer une condamnation incisive. Votre autorité en tant que membre du corps professoral s'accompagne de la responsabilité de traiter respectueusement les étudiants en toute occasion. Si votre mes sage est teinté d'affectivité et camoufle une intention cachée de punition, de menace, d'intimidation ou d'affirmation de votre statut au détriment de l'étudiant, mieux vaut vous abstenir.

$N$ os étudiants sont parfois, avec plus ou moinsd'intensité, désappointés lorsque leurs efforts ne produisent pas les résultats qu'ils espéraient obtenir. Endé, dans son article, ne fournit pas de suggestion sur la façon de se comporter face aux réponses émotives de nos étudiants. Q uelquesuns vont nier la véracité des observations, rejeter le blâme sur d'autres, chercher d'excellentes raisons expliquant pourquoi leur travail n'est pas idéal ou même réagir avec colère envers l'enseignant qui donne le feedback. Si les principes d'un feedback efficace sont suivis, ces tactiques 


\section{Références}

\section{1- Vous êtes la personne appropriée pour offrir du} feedback si :

- vous avez observél'étudiant,

- vous possédez l'expertise requise dans le domaine,

- vous êtes responsable de l'éval uation de l'étudiant.

\section{2- Vos messages de feedback devraient :}

- se référer à des comportements observables,

- se référer à des comportements remédiables,

- être basés sur de l'information valide et complète,

- offrir du feedback aussi bien positif que négatif,

- être libres de toute intention préconçue,

- être spécifiques.

\section{3- Votre feedback devrait être offert :}

- sur une base fréquente et régulière,

- à un moment approprié,

- après que les émotions sont retombées,

- dansun environnement privé et serein.

\section{4- Lorsque vous offrez du feedback, vous devriez :}

- impliquer l'étudiant,

- vous assurer d'un accord quant aux buts et aux objectifs,

- limiter sa quantité,

- donner un feedback équilibré,

- utiliser un langage objectif, qui ne porte pas

de jugement à l'égard des personnes,

- identifier explicitement les commentaires subjectifs,

- offrir des suggestions,

- vérifier la compréhension du feedback.

\section{5- Pour amortir l'impact émotionnel du feedback, vous devriez:}

- Savoir identifier la présence d'une réponse émotive,

- M ontrer votre respect de l'apprenant,

- Être respectueux de ses sentiments.

\section{6- Pour approfondir votre intérêt envers le feed back, vous devriez :}

- rechercher des ateliers,

- solliciter du feedback sur votre feedback,

- vous entraîner,

- partager vos connaissances, vos habiletés et votre enthousiasme,

- continuer à en donner. défensives devrai ent être limitées. En fait, le feedback peut même intégrer les émotions négatives de l'apprenant, les mettre en perspectiveet ultimement les réorienter vers des résultats positifs.

Lorsque l'étudiant devient résistant ou défensif, certaines techniques permettent malgré tout de tirer le meilleur parti de la situation. Commencez par identifier explicitement l'inconfort apparent du résident et par lui proposer d'en explorer le motif ensemble. M ettez l'accent sur les points positifs, sans esquiver les éléments négatifs. $D$ écomposez les observations qui posent problème en explicitant leurs différents éléments et encouragez les résdents à s'approprier une part du problème tout au moins. $N$ égociez l'acceptation de petites zones de contentieux. Prenez du temps pour examiner les différents éléments individuellement puis regroupez-les et laissez la responsabilité à qui elle appartient.

\section{Comment puis-je m'améliorer pour mieux donner du feedback?}

N ous assistons, dans le monde universitaire, à un d'intérêt croissant voire à un enthousiasmeà propos de l'incorporation du feedback efficace dans nos activités d'enseignement. Peut-être est-ce parce qu'en tant que cliniciensenseignants nous nous rendons souvent compte que la nécessité de délivrer davantage de « bon » feedback se heurte à notre impréparation pour en fournir. Peu d'enseignants reçoivent uneformation formelle sur le feedback, de sorte que notre inconfort est très compréhensible. Pour satisfaire ces besoins de formation perçus, des ateliers portant sur l'art de donner du feedback ont proliféré dans les milieux universitaires ${ }^{18,19}$. Lors de ces ateliers dispensés sous forme de sessions interactives, les participants reçoivent habituellement des informations sur le feedback, sont ensuiteamenés à exercer leurs habi letés puis reçoivent eux-mêmes un feedback donné par leurs pairs. Les organisateurs de ces ateliers pensent généralement que les participants assimilent rapidement les principes généraux du feedback efficace durant les parties interactives de l'atelier. Les évaluations faites par les participants confortent cette hypothèse ${ }^{18,20}$. Les observateurs externes notent éga lement que les enseignants formés donnent un meilleur feedback mais la différence notée chez ceux qui ont reçu une formation spécifique est cependant moindre que celle qui est perçuepar les partici pants des ateliers ${ }^{19,21}$.

La formation professorale à l'égard du feedback représente un domaine de recherche potentiellement riche, mais peu 
exploré. Le développement de questions de recherche appropriées pour évaluer les bénéfices des activités de formation professorale en matière de feedback constitue un défi en lui-même. Le premier obstacle à franchir concerne le choix des critères d'efficacité à appliquer pour ces ateliers. D evrions-nous demander aux participants s'ils ont amélioré leur façon de donner du feedback ? D evrionsnous interroger ceux qui reçoivent leur feedback ? Lescommentaires anecdotiques, les taux de satisfaction et les questionnaires d'auto-évaluation sont intéressants mais subjectifs et ils ne fournissent pas des preuves incontestables d'efficacité. Q uels outils devons nous utiliser ? Les évaluations standardisées en situation d'enseignement simulée constituent des mesures objectives. Les enregistre ments de rencontres qui sont ensuite cotées par un ou plusieurs évaluateurs sur des grilles préélaborées sont égale ment des moyens de mesure intéressants. Ces deux types

\section{Références}

1. Weiner N. The human use of human being. In: Cybernetics and Society, Boston, Houghton M ifflin C0, 1950, p 71.

2. Klen RH, Babineau R. Eval uating the competence of trainees: It's nothing personal. Am J Psychiatry 1974 ; 131 : 788-91.

3. H anson PG. Giving feedback: An interpersonal skill. In : The 1975 Annual H andbook for Group Facilitators, San Diego (CA), University Associates Publishers, 1975.

4. Freidson E : Preface in : Bucher R, Stelling JD (eds) : Becoming Professional, Beverly H ills (CA), Sage Publications Inc 1977 : 12.

5. NadlerD A : Feedback and O rganization D evelopment; $U$ ing $D$ ataBased M ethods. Reading M ass Addi son-Wesey Pub Co Inc, 1977.

6. EndeJ. Feedback in Medical Education. JAM A $1983 ; 250: 771$ 81.

7. Wright SM, Kern DE, Kolodner K, H oward DM, Brancati FL. Attributes of excellent attending physician role models. N Engl J M ed 1998 ; 339 : 1986-93.

8. Irby D M. What clinical teachers in medicine need to know. Acad M ed $1994 ; 69: 333-42$.

9. Ilgen DR, Peterson RB, M artin BA, Boeschen DA. Supervisor and subordinate reactions to performance appraisal sessions. Organizational Behavior and Human Performance $1981 ; 28$ : 311-30.

10. I saacson JH, Posk LK, Litaker DG, H alperin AK. Reident perceptions of the evaluation process. Society of General Internal M edicine. J Gen Intern M ed. 1995 : 10(suppl) : 89. de mesures basées sur la performance sont cependant oné reux et exigent du temps pour être réal isées. La plupart de ces mesures présentent également l'inconvénient d'être sujettes au biais d'apprentissage « prétest-postest ». II reste encore beaucoup de choses à apprendre sur la façon d'administrer un feedback efficace et sur les méthodes optimales pour faire acquérir ces habiletés aux enseignants des milieux cliniques. Les personnes intéressées par la recherche en formation professorale devraient entreprendre des projets de recherche sur ces thèmes.

En dépit de la relative raretéde données probantes concernant le feedback efficace, l'atelier apparaît comme un des moyens les plus accessibles pour faciliter l'acquisition des habiletés de feedback. Les éducateurs intéressés sont encouragés à participer à des ateliers portant sur cethème au sein de leur faculté, de leur association de spécialité ou au sein des forums d'éducation médicale.

11. M anzoni JF. A better way to deliver bad news. Harvard Business Review. 2002 (Sept) : 114-9.

12. Buron RJ, M CD onald-M ann D. Giving feedback to subordinates I deasinto action guidebooks. Centrefor Creative Leadership. 2001.

13. Spickard A. Words hard to say and hard to hear. "M ay I give you some feedback."] Gen Int M ed. 1998 ; 13 : 142-3.

14. Hewson M G Little M L. Giving Feedback in M edical Education : Verification of Recommended Techniques I Gen Intern M ed 1998; $13: 111-6$.

15. Latting JK. Giving Corrective Feedback : A D ecisional Analysis. Social Work $1992 ; 37$ : 424-30.

16. Stritter FT, Baker RM, Shahady EJ : Clinical Instruction. In : $M$ C $G$ aghieWC \& F rey JJ, H andbook for the A cademic Physician. New York : Springer-Verlag, 1986.

17.Weitzel SR : F eedback That Works: How to Build and D eliver Your Message (An IdeasInto Action Guidebook). G reensboro N C : Center For Creative Leadership, 2000.

18. O'Brien HV. Unpublished Data. 1999.

19. Stone S, M azor K, D evaney-O'N eil S, Starr S, Ferguson W, Wellman S, Jacobson E, Hatern D'S, Q uirk M. D evelopment and implementation of an objectivestructured teaching exercise(OSTE) to evaluate improvement in feedback skillsfollowing a faculty deve lopment workshop. Teach Learn M ed $2003 ; 15$ : 7-13.

20. Brukner H, Altkorn DL, Cook S, Q uinn M T, M cN abb WL : Giving effective feedback to medical sudents: a workshop for faculty and house staff. Med Teach $1999 ; 21: 161-5$.

21. D unnington $G L, D$ aRosa D . A prospectiverandomized trial of a resdentsasteacherstraining program. Acad M ed $1998 ; 73: 696-700$. 